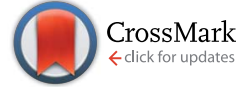

Cite this: RSC Adv., 2017, 7, 13643

\title{
Surface-engineered mesoporous silica particles with luminescent, cytocompatible and targeting properties for cancer cell imaging $\dagger$
}

\author{
Motohiro Tagaya, ${ }^{* a}$ Shigeaki Abe, $\ddagger^{\mathrm{b}}$ Satoshi Motozuka, $\$^{\mathrm{c}}$ Kota Shiba, ${ }^{\mathrm{d}}$ \\ Taro Takemura, ${ }^{\text {e }}$ Ikuo Hayashi ${ }^{f}$ and Yoshimitsu Sakaguchi ${ }^{f}$
}

To develop a novel cancer cell imaging approach, we fabricated surface-engineered mesoporous silica (MPS) particles with multi-functionalities. Specifically, mechanochemically-treated europium(III)-doped MPS (Eu:MPS) particles were prepared, and a folate $N$-hydroxysuccinimidyl ester (FA-NHS) molecule was immobilized on the particle surface as a targeting ligand for specific types of cells. With the mechanochemical treatment, the siloxane bonds were changed to show better luminescence quantum efficiency as revealed by ${ }^{29} \mathrm{Si}-\mathrm{NMR}$ and photoluminescence spectroscopy, suggesting interaction changes between the silica framework structures and the doped Eu ions. Furthermore, the mechanochemically-treated particles immobilized with FA-NHS specifically bind to the cancer cells and the subsequent uptake by the cells was in situ observed using time-lapse optical microscopy. The particles did not exhibit any toxicity in the cellular proliferation stages. The particles after the binding and uptake also showed intense luminescence from the cells at a culture time of $24 \mathrm{~h}$, demonstrating a clear imaging ability along with all the cellular shapes. Therefore, the present surface-engineered luminescent particles will be used for specific cancer targeting and imaging purposes.

Received 13th January 2017

Accepted 23rd February 2017

DOI: $10.1039 / c 7 r a 00535 k$

rsc.li/rsc-advances platforms in cell culture systems. ${ }^{2,3}$ After the discovery of mesostructured silicate, mesoporous silicas (MPSs) prepared by a supramolecular templating method possess attractive features such as well-defined and controllable pore size, large surface area, and reactive surface. ${ }^{4-6}$ The immobilization capacity of the mesopores for various functional molecules is one of the attractive features, accompanied by controlling the structural properties on a nanometer scale such as the pore and particle sizes as well as framework structures..$^{7-17}$ The MPS particles also lead to the immobilization of biofunctional molecules in the mesopore, and the surface modification for controlling the silica-molecule ${ }^{56}$ as well as silica-cell interactions has been attractive for biomedical applications. ${ }^{18-21}$

The preparation of the silica-based hybrids ${ }^{22,23}$ and the MPS particles having imaging properties for bio-function ${ }^{24-27}$ has been reported. Because of the suitable properties for such applications, highly-luminescent species of quantum dot in the mesopores have emerged for investigating the reactivity with cells as well as the incorporated heterogeneous states; $;^{28-30}$ however, heteroelements $\left(\mathrm{Cd}^{2+}, \mathrm{Hg}^{2+}, \mathrm{Se}^{2+}\right.$, etc. $)$ or guest species for the luminescence in the most cases cause often toxic effects on the cell viability. For in vivo applications, some issues of rapid color degradation, excitation by phototoxic light, and use of cytotoxic elements should be solved. As a further strategy, the external surface and silica frameworks of MPS should be functionalized as the targeting and imaging, respectively. In our previous reports, luminescent europium(III) $\left(\mathrm{Eu}^{3+}\right)$-doped 
mesoporous silica (Eu:MPS) was synthesized, ${ }^{31,32}$ and the $\mathrm{Eu}^{3+}$ doped silica frameworks enabled the accommodation of various functional molecules on the internal pore as well as the immobilization with targeting ligands only on the external surfaces, ${ }^{33-35}$ suggesting the possibility of specific cellular binding and uptake systems. The enhanced photofunctions in the particle with mesoporous structures for biomedical applications and effective immobilization on the surfaces have not yet been investigated.

To improve the environmental sites of doped luminescent species such as $\mathrm{Eu}^{3+}$ ions, the activated silica surfaces of the mesopores can be mechanochemically prepared; the nearsurface silica structure produces the densely O-rich states. We have reported the preparation of functionalized metal oxides by means of a mechanochemical surface treatment, ${ }^{36-38}$ which is a very powerful tool to modify the solid state functions (e.g., reactivity with heteroatoms). The goal to investigate the mechanochemical reactions is to find the major factors that determine the favorable photo-excited states of the doped luminescent species as well as to reveal the possibilities of regulating the silica framework in the mesostructures. ${ }^{39,40}$ For these reasons, we have focused on the controllable silica framework structures which can be interpreted as the formation changes of the siloxane and silanol groups with unpaired electrons generated, ${ }^{\mathbf{4 1 , 4 2}}$ and they are expected to provide novel activation properties by the $\mathrm{O}$ atoms to effectively improve the doped luminescent heteroatoms in the inner silica and subsequent functional molecular immobilization on the outer silica surfaces.

In this study, the luminescent Eu:MPS particles were synthesized in a sol-gel process, which enables $\mathrm{Eu}^{3+}$ ions to be homogeneously dissolved to prevent aggregation in the silica frameworks. The Eu:MPS particles were mechanochemically activated to control the photoluminescence properties through the modification of silica framework structures as shown in

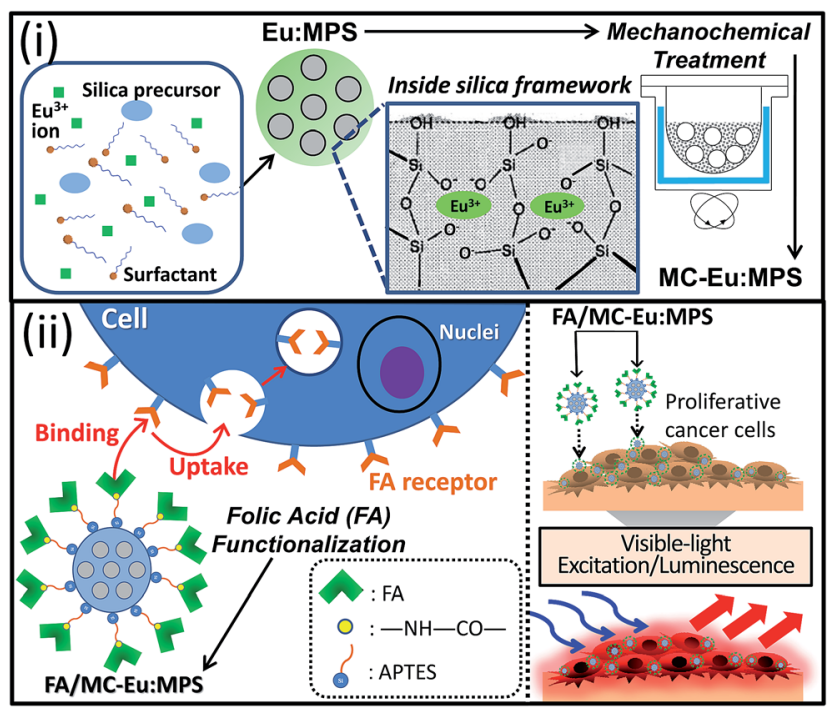

Scheme 1 Illustration of the present surface-engineering strategies: (i) preparation of the MC-Eu:MPS particles and (ii) imaging of the proliferated cancer cells by the immobilization of folic acid (FA) molecule, the uptakes by the cells, and the visible-light excitation and luminescence.
Scheme 1. Then, a folic acid (FA) molecule, which has been known as a targeting reagent for cancer cells, ${ }^{43}$ was covalently immobilized on the particles by a mediation of 3-aminopropyltriethoxysilane (APTES). Furthermore, in vitro cytotoxicity and imaging ability of the FA-functionalized particles for MG-63 and HeLa cancer cells were investigated. The effective targeting to the cells based on the surface-engineered luminescent particles was demonstrated under the visible-light excitation/ luminescence system.

\section{Experimental}

\section{Synthesis of the Eu:MPS particles}

$\mathrm{Eu}^{3+}$-doped MPS particles were synthesized according to our previous reports. ${ }^{31,32}$ Briefly, $1.0 \mathrm{~g}(2.75 \mathrm{mmol})$ of cetyltrimethylammoniumbromide (CTAB: Wako Co., Ltd.), $225 \mathrm{~g}$ of ultrapure water and $3.5 \mathrm{~mL}$ of $2.0 \mathrm{M}$ sodium hydroxide $(\mathrm{NaOH}$ : Wako Co., Ltd.) solution were stirred at $80{ }^{\circ} \mathrm{C}$ for $30 \mathrm{~min}$. Then, $5.515 \mathrm{~mL}$ (24.7 mmol) of tetraethoxysilane (TEOS: Tokyo Kasei Industries Co., Ltd.) and $15 \mathrm{~mL}$ of an $\mathrm{Eu}^{3+}$ aqueous solution containing $0.452 \mathrm{~g}$ of $\mathrm{EuCl}_{3} \cdot 6 \mathrm{H}_{2} \mathrm{O}$ (Kanto Chemical Co., Ltd.) at the initial molar concentration of $\mathrm{Eu}^{3+}$ to $\mathrm{Si}$ of $5.0 \mathrm{~mol} \%$ were added under stirring at $80{ }^{\circ} \mathrm{C}$ for $2 \mathrm{~h}$. The resultant turbid solution was filtered, and the solid product was washed with ultrapure water and ethanol, followed by drying in vacuum for $4 \mathrm{~h}$. The CTAB template was completely removed by the calcination at $550{ }^{\circ} \mathrm{C}$ for $6 \mathrm{~h}$. The final product was abbreviated as Eu:MPS. The amount of doped $\mathrm{Eu}^{3+}$ was measured by an inductively coupled plasma optical emission spectrometer (ICPOES: Leeman Labs, INC U.S.A., Prodigy ICP), suggesting that the molar concentration of $\mathrm{Eu}$ to Si was $5.4 \pm 2.1 \mathrm{~mol} \%$.

\section{Mechanochemical milling treatment of the Eu:MPS particles}

A stainless steel vessel mounted on a centrifugal equipment (Nisshin Giken Co., Ltd., NEV-MA-8) was swung at the rate of $40.2 \mathrm{rad} \mathrm{s}^{-1}$ for $2 \mathrm{~h}$ as shown in the Fig. S1(a and b), ESI. $\uparrow^{39}$ Twenty bearing steel balls (SUJ-2) with the different diameters of 4.0, 6.4 and $9.8 \mathrm{~mm}$ were used as the milling media for $0.1 \mathrm{~g}$ of the Eu:MPS particles. The balls move in a circular path against the inside wall of the vessel pressed against the wall by the centrifugal force. When the balls compress the trapped samples, the kinetic energy transfer from the medium ball to the particles occurs. The induced forces by the mechanochemical reaction was 10, 45 and $153 \mathrm{mN}$, and the resultant particles were abbreviated as MC1-Eu:MPS, MC2-Eu:MPS, and MC3-Eu:MPS, respectively. In this case, the centrifugal force exerted on the medium is classified into two forces in the Fig. S1(c), ESI; † i.e., a compression force exerted on the particles and a shear force exerted between the particles. The compression force is exerted into the depth of the particle, whereas the shear force acts only on the particle surface.

\section{Synthesis of folate $\boldsymbol{N}$-hydroxysuccinimidyl ester (FA-NHS)}

FA-NHS was prepared according to our previous reports. ${ }^{35,44}$ Briefly, $1.0 \mathrm{~g}$ of FA (FA: Wako Chemical Co., Ltd.) was dissolved in $40 \mathrm{~mL}$ of dimethyl sulfoxide (DMSO: Wako Chemical Co., 
Ltd.) containing $0.5 \mathrm{~mL}$ of triethylamine (Wako Chemical Co., Ltd.). A $0.52 \mathrm{~g}$ (2.2 equiv.) of $N$-hydroxysuccinimide (NHS: Wako Chemical Co., Ltd.) and $0.50 \mathrm{~g}$ (1.1 equiv.) of dicyclohexylcarbodiimide (DCC: Wako Chemical Co., Ltd.) were added to the solution under stirring in the dark for $18 \mathrm{~h}$. The turbid solution was filtered to remove the precipitated by-products. The colature was evaporated in vacuum to remove triethylamine. The resultant solution clearly contains FA-NHS, which was confirmed by a ${ }^{1} \mathrm{H}-\mathrm{NMR}$ analysis.

\section{Immobilizations of APTES and FA-NHS on the particles}

According to our primary report, ${ }^{33}$ the APTES immobilization was conducted by stirring the Eu:MPS $(250 \mathrm{mg}$ ) dispersed into $12 \mathrm{~mL}$ of an $\mathrm{HCl}$ aqueous solution with the mixed solution of APTES $(0.78 \mathrm{~mL})$ and ethanol $(5 \mathrm{~mL})$ at $37{ }^{\circ} \mathrm{C}$ for $1 \mathrm{~h}$. The suspension was filtered, and the solid product was washed by ethanol, followed by drying in vacuum to obtain the APTESimmobilized Eu:MPS (APTES/MC-Eu:MPS). Then, FA-NHS immobilization was conducted by stirring the APTES-Eu:MPS (150 mg) dispersed in $25 \mathrm{~mL}$ of a phosphate buffer (PB: $\mathrm{pH}=$ 7.0, phosphate ion concentration of $50 \mathrm{mM}$ ) and $12 \mathrm{~mL}$ of a DMSO solution containing the FA-NHS at $37{ }^{\circ} \mathrm{C}$. The solid product was collected by filtration, washed with ultrapure water and dried in vacuum. The immobilization amount of FA-NHS was controlled by three different initial concentrations; the equilibrium concentrations in the solution were $0.257,1.24$ and $2.99 \mathrm{mM}$. The ideal amount of the FA-NHS immobilized on each product was 39, 61 and $83 \mu \mathrm{mol}$ (g of APTES-Eu:MPS) ${ }^{-1}$, which were abbreviated as FA1/, FA2/ and FA3/MC-Eu:MPS, respectively. The actual amount was photospectroscopically estimated to be $38.9 \mu \mathrm{mol} \mathrm{g}^{-1}$ for FA1-Eu:MPS, $60.8 \mu \mathrm{mol} \mathrm{g}^{-1}$ for FA2Eu:MPS and $83.0 \mu \mathrm{mol} \mathrm{g}^{-1}$ for FA3-Eu:MPS. Taking account of the monomeric molecular area of $2.6 \mathrm{~nm}^{2}$, the surface occupation rate of FA molecule is less than $100 \%$, indicating the dispersion states among the FA molecular interactions without forming the aggregation.

\section{Characterization of the particles}

The infrared spectra were recorded on a Fourier transform infrared spectrometer (FT-IR: JASCO Co., Ltd., FT/IR-4600ST). All the FT-IR spectra were measured with a KBr pellet containing a small amount of sample powder. The weight ratio of sample to $\mathrm{KBr}$ was 10 . All the spectra were recorded after subtracting a background spectrum of pristine $\mathrm{KBr}$. The measurement range, sampling time and resolution were $4000-500 \mathrm{~cm}^{-1}$, 100 and $2.0 \mathrm{~cm}^{-1}$, respectively. The X-ray diffraction (XRD) patterns were recorded by a Smart Lab (RIGAKU Co., Ltd.) using monochromatized CuK $\alpha$ radiation. The solid-state ${ }^{29} \mathrm{Si} \mathrm{DD} / \mathrm{MAS}$ NMR (solid-state) spectra, which directly excite the Si atoms to exhibit the high reliability of the spectra, were recorded on a Bruker Avance 300wbs spectrometer with a $7 \mathrm{~mm}$ rotor (5k $\mathrm{rpm}$ ) at the resonance frequency of $59.62 \mathrm{MHz}$, $4.5 \mathrm{~ms}$ pulse and $60 \mathrm{~s}$ delay time. The chemical shifts for the signals were determined by that of hexamethylcyclotrisiloxane as an external standard at $-9.55 \mathrm{ppm}$. The ratios of the detected peak areas in the spectra were calculated. The ordered nanostructures of the particles were observed using a transmission electron microscope (TEM: JEOL Co., Ltd., JEM-1400). The samples were dropcast on a carbon-coated $\mathrm{Cu}$ grid and the grid was fully dried before the measurements. The nitrogen adsorption and desorption isotherms were measured at $-196^{\circ} \mathrm{C}$ by a BELSORPmini II instrument (Microtrac/BEL Co., Ltd.). Prior to the measurement, the samples were degassed under vacuum at $120{ }^{\circ} \mathrm{C}$ for $4 \mathrm{~h}$. The surface area was evaluated by the BrunauerEmmett-Teller (BET) method. ${ }^{51}$ The APTES and FA molecular surface occupations were calculated on the basis of the molecular areas of $0.38 \mathrm{~nm}^{2}$ and $1.65 \mathrm{~nm}^{2}$, respectively, which were verified by a molecular drawing software (CS Chem3D Pro). Taking into account the APTES and FA molecular sizes, the pore size of Eu:MPS (approx. 2.4-2.7 nm) is large enough to accommodate the APTES into the mesopores; the internal mesopore size becomes approx. $0.5 \mathrm{~nm}$ after the immobilization of monolayer APTES. Then, the FA can no longer be incorporated into the mesopores, indicating the FA functionalization only on the external surfaces as shown in the Scheme S1, ESI. $\dagger^{\mathbf{3 5}}$

The photoluminescence properties were evaluated by a photoluminescence spectroscopy and microscopy. The excitation and luminescence spectra were also recorded by a FP-8500 spectrophotometer (JASCO Co., Ltd.) with the monitored luminescence at $611 \mathrm{~nm}$ and excitation wavelength at $464 \mathrm{~nm}$, respectively, at room temperature (atmosphere: air, excitationslit/detection-slit: $2 \mathrm{~nm} / 2 \mathrm{~nm}$, measure time: $0.1 \mathrm{~s}$, step width: $1.0 \mathrm{~nm}$, light cut filter on the detection side: $\left.\lambda_{\text {cut }}<515 \mathrm{~nm}\right)$. The luminescence intensities centered at the top of 591 and $611 \mathrm{~nm}$, which are abbreviated as $I_{591}$ and $I_{611}$, were calculated by the integrated intensity areas in the range of 580-600 $\mathrm{nm}$ (assigned to ${ }^{5} \mathrm{D}_{0} \rightarrow{ }^{7} \mathrm{~F}_{1}$ of $\mathrm{Eu}^{3+}$ ) and $600-640 \mathrm{~nm}$ (assigned to ${ }^{5} \mathrm{D}_{0} \rightarrow{ }^{7} \mathrm{~F}_{1}$ of $\mathrm{Eu}^{3+}$ ) based on the Voigt-function fitting at the peak tops, and the ratios of $I_{611}$ to $I_{550}\left(I_{611} / I_{550}\right)$ were calculated. The internal quantum efficiency $\left(\eta_{\text {int }}\right)$ was measured by the above spectrophotometer using an integrating sphere (internal diameter: 60 $\mathrm{mm}$ ). The incident, scattering, and luminescence light intensity spectra were measured. Their integrated peak intensities were calculated and abbreviated as $I_{0}, I_{1}$ and $I_{2}$, respectively, as shown in the Scheme S2, ESI. $\dagger$ Thus, $\eta_{\text {int }}$ was calculated by the following eqn (1).

$$
\eta_{\text {int }}=I_{2} /\left(I_{0}-I_{1}\right) \times 100
$$

The decay kinetics was also recorded by the above spectrophotometer with the same excitation and luminescence wavelength, which can be explained with an exponential function. From a Xe flash lamp $(150 \mathrm{~W})$, the decay of the luminescence intensity was detected. The decay plot time was $0.05 \mathrm{~ms}$ using a successive rotation chopper. The decay time was calculated by a numerical fitting to an exponential function based on the eqn (2).

$$
I(t)=I(0) \exp (-t / \tau)
$$

where $I(t)$ and $I(0)$ are the luminescence intensity at time $(t)$ and starting point $(t=0)$, and $\tau$ is luminescence life time in the decay process. The fluorescent microscope images were 
obtained using a luminescence microscope (OLYMPUS Co., Ltd., CKX41N-FL, excitation wavelength: 380-420 nm, exposure time: $1500 \mathrm{~ms}$, sensitivity: 20).

\section{Cell culture}

Human Osteosarcoma MG-63 cells (300441-SF) and Human (HeLa) cervical carcinoma cells (RCB0007) as the cell lines, which were provided by Cell Lines Service and Riken BioResource Center, respectively, were separately cultured in a plastic cell culture flask with the area of $75 \mathrm{~cm}^{2}$ (BD Bioscience, USA.). The flask contained $15 \mathrm{~mL}$ of fetal bovine serum (FBS: product no. 12603C, lot no. 6D0975, SAFC Bioscience Co., Ltd.) dispersed into alpha minimum essential medium ( $\alpha$ MEM: Invitrogen Co., Ltd.) for MG-63 cells and Dulbecco's minimum essential medium (DMEM: Invitrogen Co., Ltd.) for HeLa cells at the concentration of 10 vol\% of FBS (10\% FBS/MEM). The two types of cells were used as the cervical cancer model in this study. The cells are known to express the folate receptor- $\alpha$ (FR$\alpha),{ }^{45}$ which can effectively bind with FA molecules. The cells were incubated at $37{ }^{\circ} \mathrm{C}$ in a humidified atmosphere of $\mathrm{CO}_{2}$ (5\%), and subcultured using $1 \mathrm{~mL}$ of $0.05 \mathrm{w} / \mathrm{v} \%$ trypsin- $0.053 \mathrm{M}$ ethylenediaminetetraacetate (trypsin-EDTA: No. 204-16935, Wako Co., Ltd.). After being washed with $15 \mathrm{~mL}$ of PBS and treated with $1 \mathrm{~mL}$ of the trypsin-EDTA for $5 \mathrm{~min}$ at $37^{\circ} \mathrm{C}$, the cells were homogeneously dispersed in $15 \mathrm{~mL}$ of PBS, separated by centrifugation ( $2000 \mathrm{rpm}, 2 \mathrm{~min}$ ), and dispersed in $15 \mathrm{~mL}$ of $10 \%$ FBS/MEM again. The number of cells in the suspension was counted and adjusted at the desired seeding densities in the following procedure.

\section{Evaluation of cytocompatibility and uptake properties by adding FA/MC-Eu:MPS particles}

The cell suspensions were added and cultured at the seeding area density of 8000 cells per $\mathrm{cm}^{2}$ on a poly(styrene) culture well plate (TCPS: BD Falcon ${ }^{\mathrm{TM}}$ Co., Ltd.) for evaluating the cell viability, and then cultured. According to the established procedures, ${ }^{35,46,47}$ the cell viability was evaluated using the CellTiter-Glo® luminescent cell viability assay (CellTiter-Glo assay kit, Promega Co., Ltd., U.S.A.) for MG-63 cells ${ }^{46}$ and MTT assay method by 3-(4,5-dimethylthiazol-2-yl)-2,5diphenyltetrazolium bromide (Cayman Chemical Co., Ltd., MTT reagent) for HeLa cells. ${ }^{35,47}$ At the culture time of $4 \mathrm{~h}$, the MC-Eu:MPS or FA/MC-Eu:MPS particles dispersed into 10\% FBS/MEM was added to the adhered cell surfaces. The final particle concentrations were adjusted to 0, 0.01, 0.1, 1.0, 5.0 and $10 \mathrm{mg} \mathrm{mL} \mathrm{m}^{-1}$ of MC3-EuMPS, FA1/MC3-Eu:MPS, FA2/MC3Eu:MPS and FA3/MC3-Eu:MPS for evaluating the MG-63 cells and 50, 100 and $200 \mathrm{mg} \mathrm{mL} \mathrm{m}^{-1}$ of MC3-EuMPS, FA2/MC3Eu:MPS and FA3/MC3-Eu:MPS particles for the HeLa cells. Then, an additional cell culture was performed. For the culture times of $24 \mathrm{~h}$ for MG-63 and $48 \mathrm{~h}$ for HeLa cells, the cell viability in the well plate was measured and compared. Before the measurements by photospectrometer, all the cell surfaces were washed with PBS twice. Then, the viability was evaluated by the absorbance of the resulting solution using a microplate spectrophotometer (Power Scan®HT, DS Pharma Biomedical Co.,
Ltd.), and the absorbance of $1 \mathrm{~mL}$ of only 10\% FBS/DMEM without adding the particles was subtracted as the blank value. The average value between ten samples was used, and the maximum absorbance in all the samples was set at $100 \%$.

At the culture times of 4, 12 and $24 \mathrm{~h}$ for the MG-63 cells, i.e. particle-reaction times of 0,8 and $20 \mathrm{~h}$ after adding the FA2/ MC3-EuMPS and FA3/MC3-Eu:MPS particles at the concentration of $10 \mathrm{mg} \mathrm{mL}^{-1}$, the particle uptake states by the cultured MG-63 cells were in situ observed at the same position by a timelapse approach, which was done every $3 \mathrm{~min}$ for $24 \mathrm{~h}$ at the Nikon imaging center at Hokkaido university. The results of the time-lapse observation were treated using the digital image analyzing software, MetaMorph (Molecular Devices, U. S. A.).

The detailed uptake states of the cultured cells with/without FA2/MC3-Eu:MPS particles were observed by a scanning electron microscopy (SEM: Hitachi S-4800, Japan). Before the SEM observation for the cellular morphologies, the cultured cells were fixed with 3.7 vol\% formaldehyde in PBS for $10 \mathrm{~min}$ at room temperature. Then, the cells were washed twice with $1 \mathrm{~mL}$ of PBS before and after formaldehyde fixation. After the fixation, the cultured cells were soaked in a series of baths comprising 1 $\mathrm{mL}$ of EtOH/ultrapure water mixtures at the concentration of 50, $60,70,80,90$ and $100 \mathrm{vol} \%$ with a soaking time of $5 \mathrm{~min}$ in each bath. The supernatant was then exchanged three times with $t$ butyl alcohol at $37{ }^{\circ} \mathrm{C}$ for $30 \mathrm{~min}$ per time, kept at $4{ }^{\circ} \mathrm{C}$ for $30 \mathrm{~min}$, and finally freeze dried for $5 \mathrm{~h}$.

\section{Evaluation of cancer cell imaging ability}

The HeLa cell suspensions were added and cultured at the seeding area density of 8000 cells per $\mathrm{cm}^{2}$ on a TCPS with glass bottom for observing the cellular reactions. At the culture time of $4 \mathrm{~h}$, the MC3-EuMPS and FA2/MC3-Eu:MPS particles dispersed into $10 \% \mathrm{FBS} / \mathrm{DMEM}$ were added to the adhered cell surfaces to adjust the final particle concentration (i.e., 50, 100 and $200 \mathrm{mg} \mathrm{mL}^{-1}$ ), and then cultured. The cultured cells were observed by a differential interference and fluorescent confocal laser scanning microscopy (Leica Co., Ltd.). Both images at the same position were merged into one image. The excitation and observation wavelengths were $465 \mathrm{~nm}$ and 550-650 $\mathrm{nm}$. At the culture time of $24 \mathrm{~h}$, the cells were clearly adhered on the TCPS and luminescence behavior was investigated. Note that the TCPS did not show any significant luminescence.

\section{Results and discussion}

\section{Preparation of MC-Eu:MPS particles}

The size of the Eu:MPS particles ranged from approx. 300$500 \mathrm{~nm}$, which is consistent with that reported in our previous study, ${ }^{32}$ and the sizes were almost same in the case with/without the mechanochemical treatments. The $\mathrm{Eu}^{3+}$ ions were located inside the structure to electrostatically interact with the environmental $\mathrm{O}$ atoms, which would prevent aggregation among the $\mathrm{Eu}^{3+}$ ions. ${ }^{32}$ In this study, the mechanochemical treatment would induce the changes in the flexible siloxane skeleton with preserving the particles sizes. 
Fig. 1(a) shows the XRD patterns of the Eu:MPS and MCEu:MPS particles, and the inset figure displays the TEM image of a Eu:NPS particle. The TEM image clearly exhibited the highly-ordered mesopore arrangements. The cylindrical pores were homogeneously formed on the particle surfaces; the pore diameter was estimated to be $2.4-2.7 \mathrm{~nm}$ and the thickness of the pore silica wall was $0.6-1.0 \mathrm{~nm}$. No guest substances in the pores were observed (also confirmed in Fig. S2(a), ESI $\dagger$ ). The MC1-Eu:MPS particles preserved the ordered nanostructures (Fig. S2(b), ESI $\dagger$ ), whereas the nanostructures of the MC2Eu:MPS and MC3-Eu:MPS particles exhibited the deteriorated arrangements and the fusion and aggregation among the particles (Fig. S2(c and d), ESI $\dagger$ ). The diffractions in the Eu:MPS are indexed as the 100, 110, 200 and 220 planes of a hexagonal structure, suggesting the ordered pore arrangements. The MC1Eu:MPS preserved the ordered structures based on the $d_{100}$ value, although the diffractions in the MC2-Eu:MPS and MC3Eu:MPS particles exhibited the deteriorated arrangements (i.e., worm-like mesopore structures). Thus, the mechanochemical treatment was successfully applied to the Eu:MPS particles without serious damages to the pores by carefully setting the treatment condition.

Fig. 1(b) shows the $\mathrm{N}_{2}$ adsorption and desorption isotherms of the Eu:MPS and MC-Eu:MPS particles. A hysteresis between the adsorption and desorption at the relative pressure of around 0.5 was observed in the Eu:MPS and MC1-Eu:MPS particles. These isotherms were categorized into typical type IV according to the IUPAC classification, indicating the existence of mesopores that were not destroyed during the mechanochemical treatment. In the MC2-Eu:MPS and MC3-Eu:MPS particles, the isotherm shapes were different as compared with those of the other particles. The Barrett-Joyner-Halenda (BJH) pore size distributions ${ }^{57}$ indicated the dilatation of pore diameter by the milling treatment and the distribution width was broadening (Fig. S3, ESI $\dagger$ ). It is thought that the multiple $\mathrm{N}_{2}$ adsorption
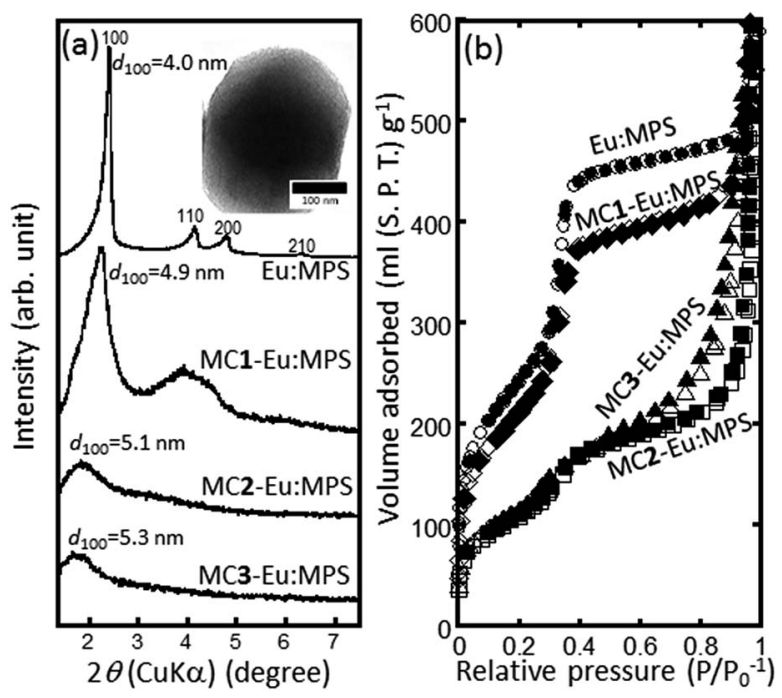

Fig. 1 (a) XRD patterns and (b) $\mathrm{N}_{2}$ adsorption (closed marks) and desorption (open marks) isotherms of the Eu:MPS and MC-Eu:MPS particles. Inset: TEM image of the Eu:MPS particle. steps would occur on the surfaces, suggesting the formation of the hierarchical surface energy structures by the fusion and aggregation between the particles.

In addition to the nanostructural changes mentioned above, the interaction changes between the particles occurred. As shown in the Fig. S1(c), $\dagger$ a medium ball is pressed against the vessel wall surface to produce a centrifugal force on a group of particles. The centrifugal force exerted on the medium is classified into two forces; i.e., a compression force exerted on the particles and a shear force exerted between the particles. The compression force is exerted into the depth of the particle, whereas the shear force acts only on the particle surface. As described elsewhere in detail, ${ }^{\mathbf{4 1}}$ the particles were repeatedly welded and then fractured by the milling force to be an equilibrium milling state. Thus, the particle size is changed due to the welding and fracturing, respectively. Consequently, it is suggested that a higher specific surface area of the MC3-Eu:MPS particles as compared with that of MC2-Eu:MPS would be attributed to the difference between the particle interactions.

As shown in Table 1, the BET surface area decreased with the mechanochemical treatment, suggesting the environmental siloxane skeleton changes for $\mathrm{Eu}^{3+}$ ions by the mechanochemical treatments.

According to our previous reports, ${ }^{\mathbf{4 1 , 4 2}}$ the metal oxide surfaces have the active $\mathrm{O}$ atoms with rich dangling bonds in the disordered $\mathrm{SiO}_{x}$. The surfaces significantly affect the attractive adsorption force and rearrange the oxide structures, suggesting the strong molecular orbital interactions between the $\mathrm{Si}-\mathrm{O}$ bonds, $\mathrm{Eu}^{3+}$ ions and FA-NHS molecules. Therefore, the mechanochemical force on the near-surface Eu:MPS would affect the luminescence properties as well as the immobilization states in the present case.

\section{Luminescence properties of the MC-Eu:MPS particles}

Fig. 2 shows the excitation and photoluminescence spectra of the MC-Eu:MPS particles. The fluorescent microscope image indicates the orange luminescence from the particles. The peaks of the MC-Eu:MPS particles are attributed to the transitions from the metastable orbital singlet state of ${ }^{5} \mathrm{D}_{0}$ to the spin-orbital states of ${ }^{7} \mathrm{~F}_{J}(J=1,2,3,4),{ }^{49}$ which specifically indicates the transitions from the ${ }^{5} \mathrm{D}_{0}$ to ${ }^{7} \mathrm{~F}_{0}$ at $577 \mathrm{~nm},{ }^{7} \mathrm{~F}_{1}$ at $591 \mathrm{~nm},{ }^{7} \mathrm{~F}_{2}$ at $611 \mathrm{~nm},{ }^{7} \mathrm{~F}_{3}$ at $654 \mathrm{~nm}$ and ${ }^{7} \mathrm{~F}_{4}$ at $700 \mathrm{~nm}$. Irrespective of the condition of the mechanochemical treatments, the spectral shapes are almost same. The luminescence of the $\mathrm{Eu}^{3+}$ doped in the silica framework was predominantly attributed to ${ }^{5} \mathrm{D}_{0} \rightarrow{ }^{7} \mathrm{~F}_{1}$ and ${ }^{5} \mathrm{D}_{0} \rightarrow{ }^{7} \mathrm{~F}_{2}$, and the ${ }^{5} \mathrm{D}_{0} \rightarrow{ }^{7} \mathrm{~F}_{2}$ intensity depended on the site environment of $\mathrm{Eu}^{3+} \cdot{ }^{32,50}$ The ratio of the

Table $1 S_{\mathrm{BET}}, \eta_{\mathrm{nt}}$ and $\tau$ of the Eu:MPS and MC-Eu:MPS particles

\begin{tabular}{llcl}
\hline & $S_{\text {BET }}\left(\mathrm{m}^{2}(\mathrm{~g} \text { of silica })^{-1}\right)$ & $\eta_{\text {int }}(\%)$ & $\tau(\mathrm{ms})$ \\
\hline Eu:MPS & 894 & 6.5 & $1.234 \pm 0.012$ \\
MC1-Eu:MPS & 793 & 6.3 & $1.052 \pm 0.016$ \\
MC2-Eu:MPS & 401 & 8.2 & $0.926 \pm 0.004$ \\
MC3-Eu:MPS & 437 & 10.1 & $0.811 \pm 0.001$
\end{tabular}




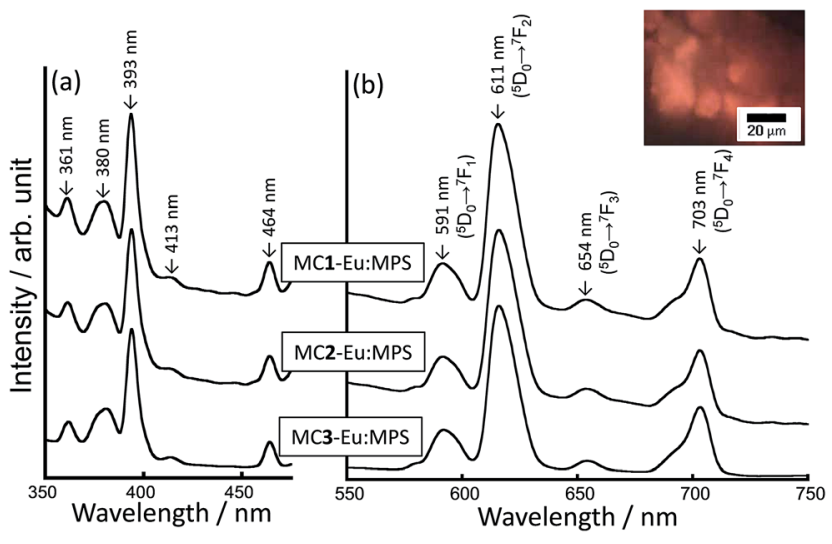

Fig. 2 (a) Excitation and (b) photoluminescence spectra of the MCEu:MPS particles treated with the different milling forces. Inset: representative fluorescence image of the MC3-Eu:MPS particles.

${ }^{5} \mathrm{D}_{0} \rightarrow{ }^{7} \mathrm{~F}_{2}$ to ${ }^{5} \mathrm{D}_{0} \rightarrow{ }^{7} \mathrm{~F}_{1}$ luminescence intensities $\left(I_{611} / I_{590}\right)$ was 5.0, 5.1 and 5.4 for MC1-Eu:MPS, MC2-Eu:MPS and MC3Eu:MPS particles, respectively, indicating the slight symmetry changes for the doped $\mathrm{Eu}^{3+}$ ions.

The stability of MPS in biological solution has been reported and the dependence of ionic-strength was pointed out. ${ }^{55} \mathrm{We}$ have reported the stability of the Eu:MPS particles in PBS, ${ }^{33}$ suggesting the importance of the FA functionalization for dispersion stability. In this study, the MC3-Eu:MPS stably dispersed in PBS (Fig. S4(a), ESI $\dagger$ ) and exhibited strong and stable photoluminescence (Fig. S4(b), ESI†). In the present case, such stability in the biological solution was due to the $\mathrm{Eu}^{3+}$ ions which are densely-confined by the silica frameworks.

Surprisingly, $h_{\text {int }}$ increased with increasing the mechanochemical force as shown in Table 1 . The $h_{\text {int }}$ based on the luminescence from ${ }^{5} \mathrm{D}_{0} \rightarrow{ }^{7} \mathrm{~F}_{2}$ is in the order of MC3-Eu:MPS > MC2-Eu:MPS > MC1-Eu:MPS. The stronger mechanochemical

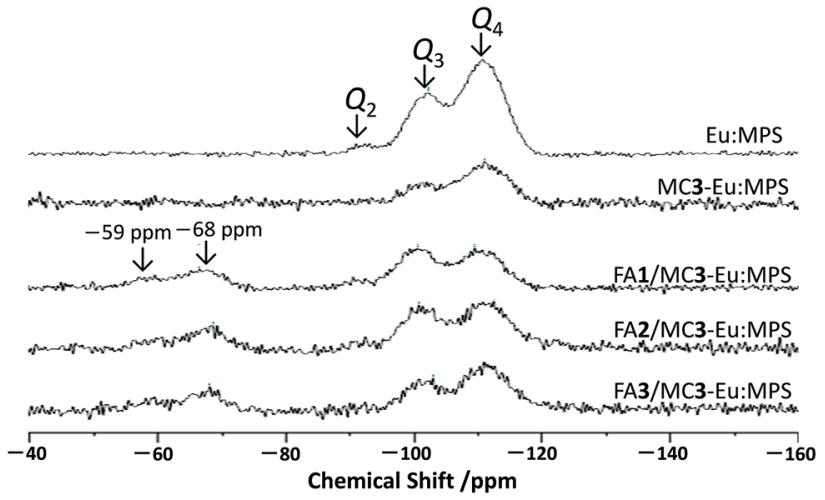

Fig. $3{ }^{29} \mathrm{Si}$ DD/MAS NMR (solid-state) spectra of each sample. force would induce the complete disruption of the mesopores as well as the segregation of europium and/or silicon oxides on the particle surfaces. In the different Eu ion concentrations (2.5-10 mol\%) to Si in the silica matrix, the $h_{\text {int }}$ value was $3.9-8.7 \%$ (ref. 35) and lower than that prepared by the mechanochemical treatment in this study. In the silica host systems, the cross relaxation among the neighboring $\mathrm{Eu}^{3+}$ ions would take place and quench the luminescence. ${ }^{52,53}$ Furthermore, the hydroxyl groups and adsorbed water molecules can also lower the luminescence efficiency of the $\mathrm{Eu}^{3+}$ through a non-radiative phonon quenching. ${ }^{54}$ These problems could be suppressed by the present mechanochemical treatment.

To understand the luminescence relaxation, the decay kinetics of the excited states was investigated as summarized in Table 1. With increasing the mechanochemical force, the $\mathrm{Eu}^{3+}$ ions could be juxtaposed to each other to enhance the cross relaxation among the neighboring $\mathrm{Eu}^{3+}$ ions to shorten $t$, even though only the silica framework exhibited the longer $t$ of the $\mathrm{Eu}^{3+}$. The relationship between the mechanochemical force and the $t$ reveals the favorable dispersion state of the $\mathrm{Eu}^{3+}$ ions in the silica frameworks. In the following experiment, the most luminescent MC3-Eu:MPS was used.

\section{${ }^{29}$ Si-MAS NMR spectral changes by the surface- functionalization}

Fig. 3 shows the ${ }^{29}$ Si-MAS NMR spectra of the particles. A few significant signals were seen and were attributed to $Q^{2}(=$ $\left.\mathrm{OSi}(\mathrm{OSi})_{2}\right), Q^{3}\left(-\mathrm{OSi}(\mathrm{OSi})_{3}\right)$ and $Q^{4}\left(\mathrm{Si}(\mathrm{OSi})_{4}\right)$ at around $-94,-101$ and $-110 \mathrm{ppm}$, respectively. The decrease in the $Q^{2}$ signal by the mechanochemical treatment indicates the decrease in the structural $\mathrm{Si}-\mathrm{OH}$ groups, implying the reaction among $\mathrm{Si}-\mathrm{OH}$ groups to form O-Si-O. It is also supported by the FT-IR spectra (Fig. S5, ESI $\dagger$ ) where the absorption bands originated from the $\mathrm{Si}-\mathrm{O}-\mathrm{Si}$ stretching mode were seen at around 1070 and 1225 $\mathrm{cm}^{-1}$. In addition, the other bands assigned to the $\mathrm{Si}-\mathrm{OH}$ stretching mode were also observed at around 795 and 960 $\mathrm{cm}^{-1}$. The broad bands centered at around 1633 and $3450 \mathrm{~cm}^{-1}$ are attributed to the $\mathrm{O}-\mathrm{H}$ stretching of the adsorbed $\mathrm{H}_{2} \mathrm{O}$ molecules. With the mechanochemical treatment, the slight increase in the bands at around 1633 and $3450 \mathrm{~cm}^{-1}$ and the disappearance of the band at $3740 \mathrm{~cm}^{-1}$ due to the structural $\mathrm{Si}-\mathrm{OH}$ groups are indicative of the increase in the adsorbed $\mathrm{H}_{2} \mathrm{O}$ and decrease in the $\mathrm{Si}-\mathrm{OH}$. Judging from these results, the nearsurface (e.g., structural $\mathrm{Si}-\mathrm{OH}$ groups) changes by the mechanochemical treatment would affect the interaction states with the $\mathrm{H}_{2} \mathrm{O}$ molecules.

After the reaction with APTES, two signals appeared at around -59 and $-68 \mathrm{ppm}$ that are due to $T^{2}\left(-\mathrm{C}-\mathrm{Si}(\mathrm{OSi})_{2}(\mathrm{OH})\right)$ and $T^{3}\left(-\mathrm{C}-\mathrm{Si}(\mathrm{OSi})_{3}\right)$, indicating successful immobilization. The relative integral intensity ratio of $Q^{3}$ to $Q^{4}\left(Q^{3} / Q^{4}\right)$ of the FA/MC3-

Table 2 Integral peak area ratios of $Q_{3} / Q_{4}$ in the ${ }^{29} \mathrm{Si} D \mathrm{D} / \mathrm{MAS}$ NMR solid-state spectra of each sample

\begin{tabular}{llllll}
\hline & Eu:MPS & MC3-Eu:MPS & FA1-Eu:MPS & FA2-Eu:MPS & FA3-Eu:MPS \\
\hline$Q_{3} / Q_{4}$ & 0.61 & 0.47 & 1.04 & 0.63
\end{tabular}


Eu:MPS particles shown in Table 2 was higher than that of the previous report on bare MCM-41 (0.62), ${ }^{48}$ suggesting the increase in the structural $\mathrm{Si}-\mathrm{OH}$ groups by the FA immobilization process. In fact, the APTES immobilization induced the decrease in specific surface area with the reaction time. This would be due to the APTES molecules strongly interact with the

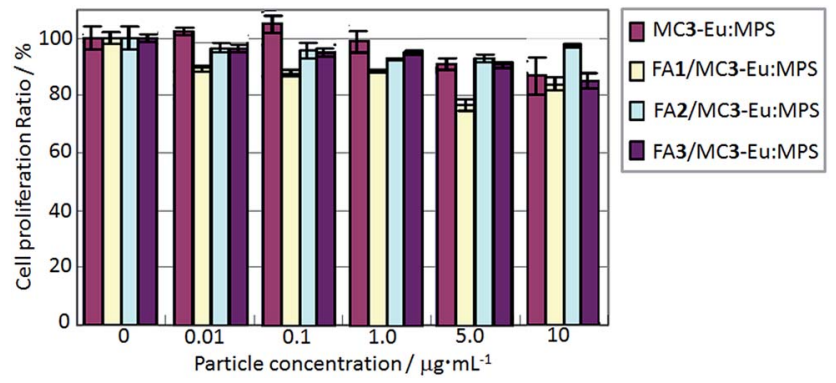

Fig. 4 The changes of MG- 63 cell proliferation ratio under the presence of the MC3-Eu:MPS, FA1/MC3-Eu:MPS, FA2/MC3-Eu:MPS and FA3/MC3-Eu:MPS particles with the different lower particle concentration $\left(0-10 \mu \mathrm{g} \mathrm{mL}^{-1}\right)$ at the culture time of $24 \mathrm{~h}$. Although this result shows no significant differences among the low particle concentrations, it suggests that the FA2/MC3-Eu:MPS and FA3/MC3-Eu:MPS particles give no cytotoxicity at the concertation of $10 \mu \mathrm{g} \mathrm{mL}^{-1}$. silica frameworks in the mesopores to change the bonding states. ${ }^{35}$ Thus, the local structural changes from $Q^{4}$ to $Q^{3}$ would occur at the mesopores. The signal broadening with the mechanochemical treatment and subsequent FA immobilization are probably due to the changes in the structural $\mathrm{Si}-\mathrm{O}$ bond distance and/or Si-O-Si angles. ${ }^{49}$ Therefore, these results clearly suggest that the silica framework structures are modified by the mechanochemical treatment and subsequent FA immobilization.

\section{Cytotoxicity and uptake properties of the FA/MC-Eu:MPS particles}

MG-63 cell proliferation ratios were investigated when they were exposed to the MC3-Eu:MPS, FA1/MC3-Eu:MPS, FA2/MC3Eu:MPS and FA3/MC3-Eu:MPS particles with the different particle concentration at the culture time of $24 \mathrm{~h}$ (Fig. 4). At the present culture time and particle concentration ranges, we visually observed that the adhered cells proliferated and maintained with their normal morphologies in the presence of the particles. It seemed that the particles bind to cellular membranes or are uptaken by the cells. Although this result indicated no significant differences among the low particle concertation of $0-10 \mu \mathrm{g} \mathrm{mL}{ }^{-1}$, the FA2/MC3-Eu:MPS and FA3/
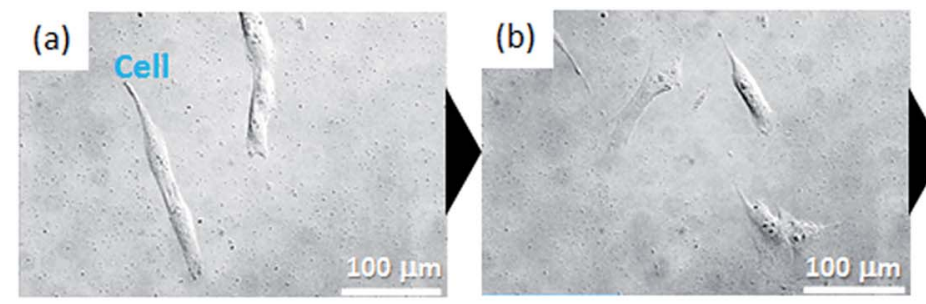

(c)

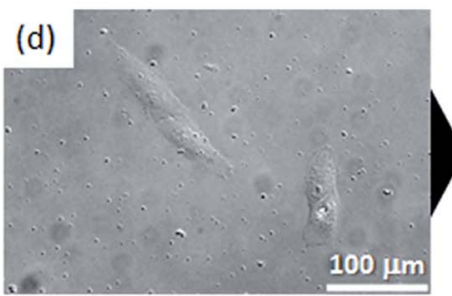

(e)

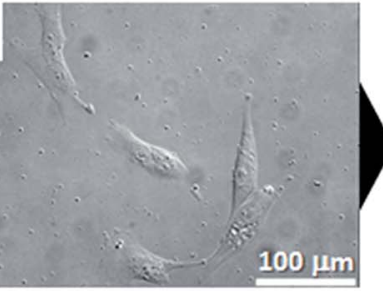

(f)
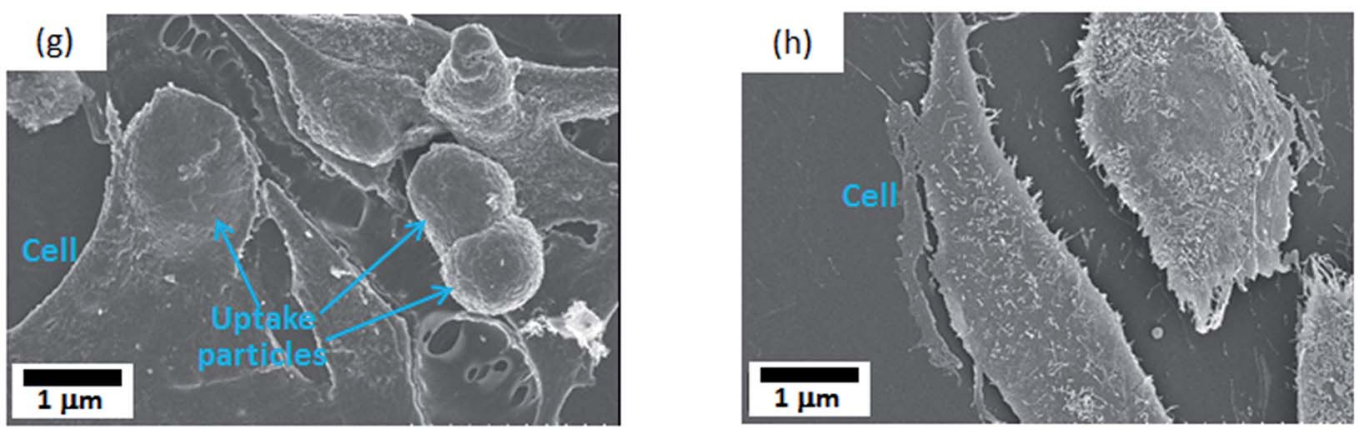

Fig. 5 (a-f) Typical time-lapse observation images exposed to (a-c) FA2/MC3-Eu:MPS and (d-f) FA3/MC3-Eu:MPS at the same observation position at the particle concentration of $10 \mu \mathrm{g} \mathrm{mL}^{-1}$ : (a and d) the MG-63 cells were going extend pseudopods to capture the particles actively (just the addition of the particles at $4 \mathrm{~h}$ ) and then ( $\mathrm{b}$ and e) aggregated them around the cell periphery at the time of $12 \mathrm{~h}$ (just $8 \mathrm{~h}$ after the addition of the particles). At the time of $24 \mathrm{~h}$ (just $20 \mathrm{~h}$ after the addition of the particles), the cells completely uptake the particles inside the cell membrane, which were also supported by (g) FE-SEM observation image after adding the FA2/MC3-Eu:MPS particles. In contrast, there is no reflection by the particulate shapes in the cell membrane on TCPS without adding the particles as shown in the FE-SEM image (h). 
MC3-Eu:MPS particles caused low cytotoxicity at the concertation of $10 \mu \mathrm{g} \mathrm{mL} \mathrm{m}^{-1}$, and were used in the following experiment.

To investigate the cellular uptake processes in more detail, the typical time-lapse observation images exposed to FA2/MC3Eu:MPS and FA3/MC3-Eu:MPS at the same observation position (the particle concentration: $10 \mu \mathrm{g} \mathrm{mL}^{-1}$ ) were shown in Fig. 5(af). The MG-63 cells were going extend pseudopods to capture the particles actively at the time of $8 \mathrm{~h}$ (just $4 \mathrm{~h}$ after the addition of the particles) in Fig. 5(a and d). Then, the particles were aggregated on the cell membrane surfaces around the periphery at the time of $12 \mathrm{~h}$ (just $8 \mathrm{~h}$ after the addition of the particles) in Fig. 5(b and e). At the time of $24 \mathrm{~h}$ (just $20 \mathrm{~h}$ after the addition of the particles), the cells would endocytose the particles. In the observation of the cell activity by the MC3-Eu:MPS particles, the cells were also going extend pseudopods to capture some particles, indicating no significant differences between MC3Eu:MPS and FA2/MC3-Eu:MPS particles. In other words, it is difficult to judge whether the cells interact with the particles in the case without washing the cell surfaces. Accordingly, we examined this point after washing the cell surfaces. As a result, the uptake was supported by the SEM observation (Fig. 5(g)). Although the cellular shapes and volumes were slightly reduced under the vacuum condition, it turns out that the cell membranes followed particle shapes to swell up by adding FA2/ MC3-Eu:MPS particles. In contrast, there was no reflection by the particulate shapes inside the cellular bodies cultured on TCPS in Fig. 5(h). As the other control (addition of the MC3Eu:MPS particles), the cell surface morphologies indicate that no evidence on the cell-particle interaction was recognized, implying the importance of the FA immobilization for the binding or uptake. Therefore, the FA/MC-Eu:MPS particles were effectively endocytosed by the cancer cells.

The cell viability changes with/without adding MC3-Eu:MPS, FA2/MC3-Eu:MPS and FA3/MC3-Eu:MPS particles at the different culture times and higher particle concentrations were shown in Fig. 6. The viability behaviors reached the confluent states at around the culture time of $50 \mathrm{~h}$. In particular, the HeLa cancer cells drastically proliferated $6 \mathrm{~h}$ after adding the FA2/

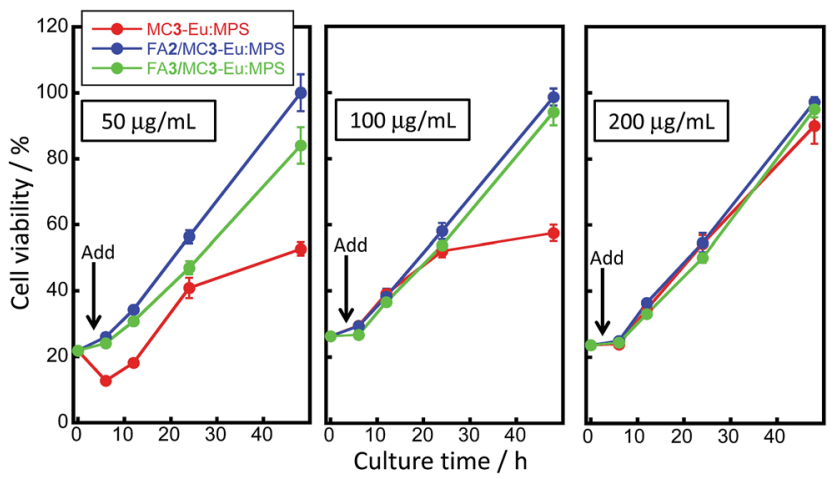

Fig. 6 Cell viability changes with/without adding MC3-Eu:MPS, FA2/ MC3-Eu:MPS and FA3/MC3-Eu:MPS particles at the different culture times and higher particle concentrations of the particles. A black arrow at the culture time of $4 \mathrm{~h}$ indicates the added point of the particles.
MC3-Eu:MPS and FA3/MC3-Eu:MPS particles. This behavior is same as that without adding the particles, indicating the reflection of the native proliferation rate and non-cytotoxicity of the FA-functionalized MC3-Eu:MPS particles. In contrast, the MC3-Eu:MPS particles exhibited the lower proliferation rate. Thus, the surface-activated MC3-Eu:MPS particles with radical species were deactivated with the FA-NHS functionalization to resultantly exhibit a good cytocompatibility. The FA2/MC3Eu:MPS particles exhibited good cytocompatibility at all the concentration and were used for the imaging experiment described in the following section.

\section{Imaging ability for cancer cells by the FA/MC-Eu:MPS particles}

Fig. 7 shows the representative composite images of both the differential interference and fluorescence images of the HeLa cells reacted with MC3-Eu:MPS and FA2/MC3-Eu:MPS particles at the different concentrations. The luminescence was easily observed by fluorescent microscopy. The significant binding and uptake by the HeLa cells were clearly recognized. The MC3Eu:MPS particles exhibited no uptake by the cells and were present outside of the cells (Fig. $7(\mathrm{a}-\mathrm{c})$ ). In contrast, the fluorescence images of the cells indicated the presence of luminescent particles located around the nuclei at the lower concentration (Fig. 7(d)). The cellular uptake would be fairly rapid and can be already observed through the cell membrane. With an increase in the particle concentration, the number of particles in the cells increased and the luminescence along with
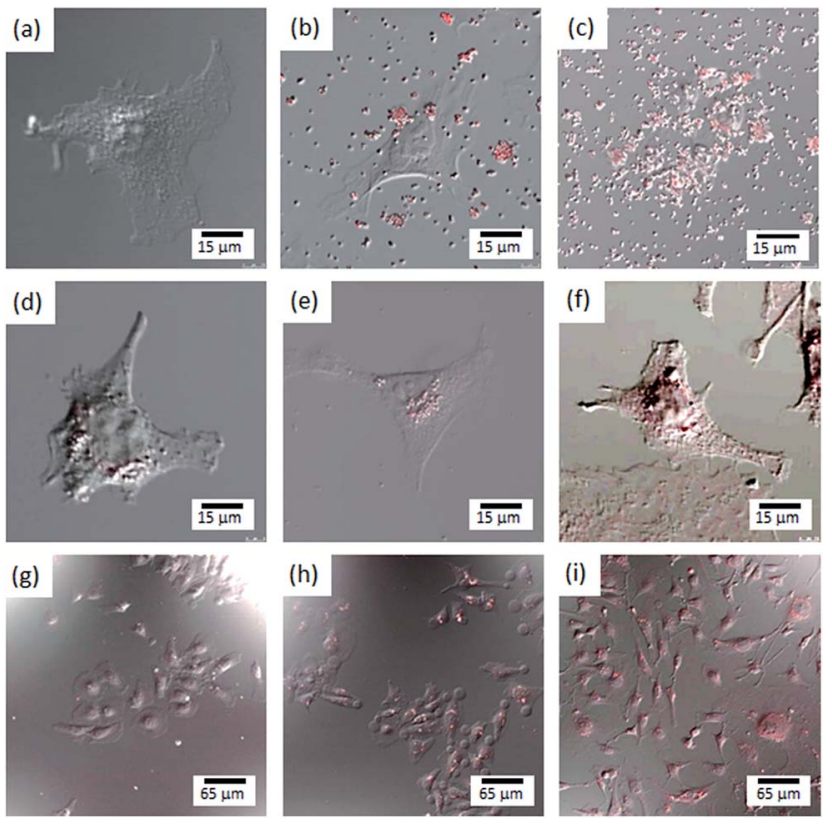

Fig. 7 Representative combined images (derived from the differential interference and fluorescence images) of the HeLa cells reacted with (a-c) MC3-Eu:MPS and (d-i) FA2/MC3-Eu:MPS particles at the different concentrations ( $\left(a, d\right.$ and g) $50 \mu \mathrm{g} \mathrm{mL}^{-1}$, (b, e and h) $100 \mu \mathrm{g}$ $\mathrm{mL}^{-1}$, (c, f and i) $\left.200 \mu \mathrm{g} \mathrm{mL}^{-1}\right)$. The $(\mathrm{g}-\mathrm{i})$ images were zoom out from the $(d-f)$ images, indicating the clear imaging ability along with all the cellular shapes. These images were taken at the culture time of $24 \mathrm{~h}$ (just $20 \mathrm{~h}$ after the addition of the particles). 
the cellular shapes were observed (Fig. 7(e and f)). It indicates that the targeted particle amount are suitable for imaging HeLa cells. Accordingly, the FA2/MC3-Eu:MPS particles were effectively taken up by the HeLa cells. Fig. $7(\mathrm{~g}-\mathrm{i})$ images were zoom out from the Fig. $7(\mathrm{~d}-\mathrm{f})$ images, indicating the clear imaging ability along with all the cellular shapes. Therefore, the FA2/ MC3-Eu:MPS particles can be readily taken up by the HeLa cells at the particle concentration of $200 \mu \mathrm{g} \mathrm{mL} \mathrm{m}^{-1}$ and will be used as potential fluorescent labels in biomedical imaging. Therefore, based on host-guest interactions, ${ }^{\mathbf{5 6}}$ the nanospaces of the particles in this study will lead to the immobilization of anticancer drug molecules in the mesopore for biomedical applications.

\section{Conclusions}

For the imaging of cancer cells, we developed a novel surfaceengineering method using the luminescent MPS particles as a model system. Specifically, the mechanochemically-treated particles were successfully prepared, and a folate $N$-hydroxysuccinimidyl ester (FA-NHS) molecule as a targeting ligand for the cells was immobilized on the particle surfaces. With the mechanochemical treatment to the particle surface, the siloxane bonding structures were changed to show better luminescence quantum efficiency as revealed by ${ }^{29} \mathrm{Si}-\mathrm{NMR}$ and photoluminescence spectroscopy, suggesting the interaction changes between the silica frameworks and the doped $\mathrm{Eu}^{3+}$ ions. We also confirmed that the mechanochemically-treated particles specifically bound to the cancer cells after immobilizing with FA-NHS and the particles did not show any toxicity in the cellular proliferation stages. The binding and uptake process by the cells were in situ observed by a time-lapse optical microscopy. The particles after the binding and uptake also showed the intense luminescence from the cells at the culture time of $24 \mathrm{~h}$, demonstrating the clear imaging ability along with all the cellular shapes. Therefore, the present surfaceengineered luminescent MPS particles will be used for specific targeting and imaging purposes for cancer cells. In future, the utilization of the drug-loaded mesostructures for theranostics will be reported by our laboratory.

\section{Acknowledgements}

This study was partially supported by JSPS KAKENHI Grant Number 26709052.

\section{Notes and references}

1 L. Brandon-Peppas and J. O. Blanchette, Adv. Drug Delivery Rev., 2004, 56, 1649-1659.

2 F. Caruso, Adv. Mater., 2001, 13, 11-22.

3 L. Hu, Z. W. Mao and C. Y. Gao, J. Mater. Chem., 2009, 19, 3108-3115.

4 T. Yanagisawa, T. Shimizu, K. Kuroda and C. Kato, Bull. Chem. Soc. Jpn., 1990, 63, 988-992.

5 C. T. Kresge, M. E. Leonowicz, W. J. Roth, J. C. Vartuli and J. S. Beck, Nature, 1992, 359, 710-712.
6 J. S. Beck, J. C. Vartuli, W. J. Roth, M. E. Leonowicz, C. T. Kresge, K. D. Schmitt, C. T.-W. Chu, D. H. Olson, E. W. Sheppard, S. B. McCullen, J. B. Higgins and J. L. J. Schlenker, J. Am. Chem. Soc., 1992, 114, 10834-10843.

7 K. B. Yoon, Chem. Rev., 1993, 93, 321-339.

8 K. Moller and T. Bein, Chem. Mater., 1998, 10, 2950-2963.

9 M. Ogawa, Annu. Rep. Prog. Chem., Sect. C: Phys. Chem., 1998, 94, 209-257.

10 A. Stein, B. J. Melde and R. C. Schroden, Adv. Mater., 2000, 12, 1403-1419.

11 B. J. Scott, G. Wirnsberger and G. D. Stucky, Chem. Mater., 2001, 13, 3140-3150.

12 H. Furukawa, T. Watanabe and K. Kuroda, Chem. Commun., 2001, 2002-2003.

13 M. Ogawa, J. Photochem. Photobiol., C, 2002, 3, 129-146.

14 N. K. Mal, M. Fujiwara and Y. Tanaka, Nature, 2003, 421, 350-353.

15 M. Tagaya and M. Ogawa, Chem. Lett., 2006, 35, 108-109.

16 M. Tagaya and M. Ogawa, Phys. Chem. Chem. Phys., 2008, 10, 6849-6855.

17 M. Tagaya, N. Hanagata and T. Kobayashi, ACS Appl. Mater. Interfaces, 2012, 4, 6169-6175.

18 M. Vallet-Regi, F. Balas and D. Arcos, Angew. Chem., Int. Ed. Engl., 2007, 46, 7548-7558.

19 M. Manzano and M. Vallet-Regi, J. Mater. Chem., 2010, 20, 5593-5604.

20 M. Tagaya, K. Kobayasi and M. Nishikawa, Mater. Lett., 2016, 164, 651-654.

21 M. Tagaya, $R S C$ Adv., 2016, 6, 14452-14456.

22 E. Ruiz-Hitzky, P. Aranda, M. Darder and M. Ogawa, Chem. Soc. Rev., 2011, 40, 801-828.

23 N. Nassif and J. Livage, Chem. Soc. Rev., 2011, 40, 849-859.

24 J. L. Vivero-Escoto, I. I. Slowing, C.-W. Wu and V. S. Y. Lin, J. Am. Chem. Soc., 2009, 131, 3462-3463.

25 M. Okuda, M. Takeguchi, Y. Zhu, A. Hashimoto, N. Ogawa, M. Tagaya, S. Chen, N. Hanagata and T. Ikoma, Surf. Interface Anal., 2011, 42, 1548-1551.

26 J. M. Rosenholm, A. Meinander, E. Peuhu, R. Niemi, J. E. Eriksson, C. Sahlgren and M. Lindn, ACS Nano, 2009, 3, 197-206.

27 V. Sokolova and M. Epple, Nanoscale, 2011, 3, 1957-1962.

28 G. Bardi, M. A. Malvindi, L. Gherardini, M. Costa, P. P. Pompa, R. Cingolani and T. Pizzorusso, Biomaterials, 2010, 31, 6555-6566.

29 J. Pan, D. Wan and J. L. Gong, Chem. Commun., 2011, 47, 3442-3444.

30 Z. Y. Cheng, P. A. Ma, Z. Y. Hou, W. X. Wang, Y. L. Dai, X. F. Zhai and J. Lin, Dalton Trans., 2012, 41, 1481-1489.

31 M. Tagaya, T. Ikoma, T. Yoshioka, F. Minami and J. Tanaka, Mater. Lett., 2011, 65, 2287-2290.

32 M. Tagaya, T. Ikoma, T. Yoshioka and J. Tanaka, J. Colloid Interface Sci., 2011, 363, 456-464.

33 M. Tagaya, T. Ikoma, T. Yoshioka and J. Tanaka, Chem. Commun., 2011, 47, 8430-8432.

34 M. Tagaya, N. Hanagata, T. Ikoma, T. Kobayashi, K. Shiba, T. Yoshioka and J. Tanaka, Key Eng. Mater., 2013, 529-530. 
35 M. Tagaya, T. Ikoma, Z. Xu and J. Tanaka, Inorg. Chem., 2014, 53, 6817-6827.

36 S. Motozuka, M. Tagaya, Y. Hotta, M. Morinaga, T. Ikoma, T. Honma, T. Daimon and J. Tanaka, Ind. Eng. Chem. Res., 2013, 52, 2182-2189.

37 S. Motozuka, M. Tagaya, K. Shiba, Z. Xu, N. Nishikawa, T. Yoshioka, T. Ikoma and J. Tanaka, Ind. Eng. Chem. Res., 2014, 53, 16736-16753.

38 S. Motozuka, M. Tagaya, N. Ogawa, K. Fukui, M. Nishikawa, K. Shiba, T. Uehara and T. Kobayashi, Solid State Commun., 2014, 190, 28-32.

39 M. Tagaya, S. Motozuka, T. Kobayashi, T. Ikoma and J. Tanaka, J. Mater. Chem., 2012, 22, 18741-18743.

40 M. Tagaya, S. Motozuka, T. Kobayashi, T. Ikoma and J. Tanaka, Ind. Eng. Chem. Res., 2012, 51, 11294-11300.

41 S. Motozuka, M. Tagaya, H. Nishiyama, M. Nishikawa, T. Ikoma, T. Yoshioka, S. Samitsu and J. Tanaka, J. Phys. Chem. C, 2013, 117, 9908-9919.

42 S. Motozuka, M. Tagaya, T. Ikoma, M. Morinaga, T. Yoshioka and J. Tanaka, J. Phys. Chem. C, 2013, 117, 16104-16118.

43 J. F. Kukowska-Latallo, K. A. Candido, Z. Y. Cao, S. S. Nigavekar, I. J. Majoros, T. P. Thomas, L. P. Balogh, M. K. Khan and J. R. Baker, Cancer Res., 2005, 65, 5317-5324. 44 J. H. van Steenis, E. M. van Maarseveen, F. J. Verbaan, R. Verrijk, D. J. A. Crommelin, G. Storm and W. E. I. Hennink, J. Controlled Release, 2003, 87, 167-176.
45 S. Y. Chan, C. J. Empig, F. J. Welte, R. F. Speck, A. Schmaljohn, J. F. Kreisberg and M. A. Goldsmith, Cell, 2001, 106, 117-126.

46 N. Iwadera, Y. Yawaka and F. Watari, Nano Biomed., 2012, 4, 57-65.

47 T. Mosmann, J. Immunol. Methods, 1983, 65, 55-63.

48 W. Chen, R. Sammynaiken and Y. Huang, J. Appl. Phys., 2000, 88, 1424-1431.

49 N. Wan, J. Xu, T. Lin, X. Zhang and L. Xu, Appl. Phys. Lett., 2008, 92, 201109-201111.

50 E. J. Nassar, K. J. Ciuffi, S. J. L. Ribeiro and Y. Messaddeq, Mater. Res., 2003, 6, 557-562.

51 S. Brunauer, P. H. Emmett and E. Teller, J. Am. Chem. Soc., 1938, 60, 309-319.

52 T. Ishizaka, R. Nozaki and Y. Kurokawa, J. Phys. Chem. Solids, 2002, 63, 613-617.

53 M. Yu, J. Lin, J. Fu, H. J. Zhang and Y. C. Han, J. Mater. Chem., 2003, 13, 1413-1419.

54 A. J. Berry and T. A. King, J. Phys. D: Appl. Phys., 1989, 22, 1419-1422.

55 I. Izquierdo-Barba, M. Colilla, M. Manzano and M. ValletRegi, Microporous Mesoporous Mater., 2010, 132, 442-452.

56 E. Aznar, M. Oroval, L. Pascual, J. R. Murguía, R. MartínezMáñez and F. Sancenón, Chem. Rev., 2016, 116, 561-718.

57 E. P. Barrett, L. G. Joyner and P. P. Halenda, J. Am. Chem. Soc., 1951, 73, 373-380. 